\title{
Acceleration of murine hepatocyte proliferation by imazalil through the activation of nuclear receptor PXR
}

\author{
Shohei Yoshimaru, Ryota Shizu, Satoshi Tsuruta, Yuto Amaike, Makoto Kano, \\ Takuomi Hosaka, Takamitsu Sasaki and Kouichi Yoshinari \\ Laboratory of Molecular Toxicology, School of Pharmaceutical Sciences, University of Shizuoka, \\ 52-1 Yada, Suruga-ku, Shizuoka 422-8526 Japan
}

(Received April 4, 2018; Accepted April 27, 2018)

\begin{abstract}
The nuclear receptor pregnane X receptor (PXR) plays a major role in the xenobiotic-induced expression of drug-metabolizing enzymes. PXR activation is also associated with several adverse events in the liver. Especially, the receptor enhances hepatocyte proliferation mediated by chemical liver tumor promoters, suggesting that exposure to PXR activators increases the risk of liver cancer. In this study, we have investigated the influences of food additives on PXR to understand their potential adverse effects when they are taken in combination with other chemical compounds. We first screened 25 food additives and related compounds for their PXR-activating ability using reporter assays in HepG2 cells expressing mouse PXR, and found that imazalil dose-dependently activated mouse PXR. Next, to investigate whether imazalil could activate mouse PXR in vivo, mice were treated with imazalil and we found that imazalil treatment increased hepatic mRNA levels of Cyp3a11, a PXR target gene. Finally, to investigate the influence of imazalil exposure on the hepatocyte proliferation induced by nuclear receptor constitutive active/androstane receptor (CAR), mice were treated with imazalil with or without mouse CAR activator TСРОВOP. Although imazalil alone did not induce hepatocyte proliferation, co-treatment with imazalil facilitated the TCPOBOP-dependent proliferation, indicated by the increases in cell proliferation marker levels, Ki-67-positive nuclei and $\mathrm{Mcm} 2 \mathrm{mRNA}$ levels. These results suggest that in mice imazalil activates PXR to enhance hepatocyte proliferation mediated by CAR-activating liver tumor promoters.
\end{abstract}

Key words: Food additive, Nuclear receptor, Pregnane X receptor, Hepatocyte proliferation, Imazalil, Enilconazole

\section{INTRODUCTION}

Food additives are used to preserve foods and make flavor, taste or appearance of the food better. There are more than 1400 food additives approved in Japan. Food additives are approved after passed the safety assessments. Quantities of each food additive contained in foods are restricted based on their acceptable daily intake (ADI), the amount that can be ingested on a daily basis over a lifetime without appreciable risk to health. Therefore, food additives are basically taken without any adverse effects in humans.

The safety assessments usually consider their exposure as a single chemical on human, and food additives are often taken with other chemicals (e.g. drugs, foods, supplements, other food additives, pesticide residues or industrial chemicals). There are some reports show- ing toxicity due to the exposure to multiple food additives. For example, both quinoline yellow and aspartame are reported to inhibit the axonal growth of mouse nerve cells. Co-exposure to these two food additives causes more harmful growth inhibition than single exposure to them (Lau et al., 2006). In K562 cells derived from chronic myeloid leukemia, the co-treatment with quercetin and curcumin, main ingredients of turmeric oleoresin, induced apoptosis by generating reactive oxygen species (ROS) more greatly than their single treatment (Mutlu Altundağ et al., 2018). While single treatment with zinc oxide nanoparticles (less than $15 \mathrm{mg} / \mathrm{L}$ ) or vitamin $\mathrm{C}$ (less than $300 \mathrm{mg} / \mathrm{L}$ ) did not cause cytotoxicity in gastric epithelial cells and neural stem cells, their co-treatment decreased the viability of these cells (Wang et al., 2014). Thus, the simultaneous exposure to several food additives may cause adverse effects that are unexpected under the

Correspondence: Kouichi Yoshinari (E-mail: yoshinari@u-shizuoka-ken.ac.jp) 
single exposure to them. It is therefore necessary to consider the synergistic effects of food additives for understanding of their potential adverse effects.

Pregnane $\mathrm{X}$ receptor (PXR) is a nuclear receptor that is activated by xenobiotics. PXR is involved in the regulation of the expression of drug-metabolizing enzymes such as CYP3A or drug transporters such as organic aniontransporting polypeptide (Staudinger et al., 2001). Ligand activated PXR is translocated to nucleus and binds to its target DNA motifs as a heterodimer with retinoid $\mathrm{X}$ receptor $\alpha(\mathrm{RXR} \alpha)$, and induces the transcription of its target genes. Thus, PXR plays an important role in the xenobiotic metabolism and excretion. In addition to this well-known function, recent studies have expanded the roles of PXR, which include the regulation of glucose or lipid metabolisms in the liver (Sui et al., 2011; Chang and Waxman, 2006; Hukkanen, 2012; Gotoh and Negishi, 2015; Spruiell et al., 2014). In addition, we have recently demonstrated that PXR activation enhances hepatocyte proliferation mediated by CAR or peroxisome proliferator-activated receptor $\alpha(\operatorname{PPAR} \alpha)$ activation: Although treatment of mice with pregnenolone $16 \alpha$-carbonitrile (PCN), a PXR agonist, alone did not induce hepatocyte proliferation, co-treatment of mice with PCN and CAR or PPAR $\alpha$ agonist showed intense hepatocyte proliferation compared to single treatment with CAR or PPAR $\alpha$ agonist (Shizu et al., 2013), which probably results from the PXR-mediated down-regulation of cell cycle suppressor genes (Shizu et al., 2016). Because CAR- and PPAR $\alpha$-dependent hepatocyte proliferation is associated with liver cancer in rodents, the exposure to PXR activators might increase the risk of liver cancer, that is, PXR-activating capability of chemicals can be a potential risk of adverse effects. Some food additives are already reported as PXR agonists such as carnosic acid, carnosol and ursolic acid in rosemary extracts or steviol, an aglycone of a steviol glycoside sweetener (Seow and Lau, 2017; Dusek et al., 2017).

In this study, we have screened food additives for their ability to activate mouse PXR (mPXR) by a reporter gene assay system and tested them in mice in vivo to verify the results of screening. At last, we have investigated the influence of co-treatment of mice with the identified PXRactivating food additive imazalil on the CAR-dependent hepatocyte proliferation to reveal whether it could have a potential to synergistically induce liver tumor promotion thorough PXR activation.

\section{MATERIALS AND METHODS}

\section{Reagents}

D-(-)-Isoascorbic acid, L-(+)-ascorbic acid, ( \pm )- $\alpha$-tocopherol, imazalil, propylene glycol, annatto, acesulfame K, saccharin sodium dihydrate, glycyrrhizic acid monoammonium salt n-hydrate, aspartame, sucralose, benzoic acid, sorbic acid, corn oil were obtained from Wako Pure Chemical Industries (Osaka, Japan). Dibutylhydroxytoluene, rebaudioside A, Food Yellow No. 4, Food Yellow No. 5, Food Red No. 3, Food Red No. 40, Food Red No. 102, Food Red No. 106, and Food Blue No. 1 were obtained from Tokyo Chemical Industry (Tokyo, Japan). (+)- $\gamma$-Tocopherol, thiabendazole, 1,4bis[2-(3,5-dichloropyridyloxy)]benzene (TCPOBOP) and PCN were obtained from Sigma-Aldrich (St. Louis, MO, USA). Stevioside was obtained from Kanto Chemical (Tokyo, Japan).

\section{Plasmid DNA}

A luciferase reporter construct was prepared by inserting five repeats of dNR1 (Goodwin et al., 1999) and eNR3A4 (Toriyabe et al., 2009) from the CYP3A4 promoter region into KpnI and NheI sites and NheI and BglII sites of pGL3-Promoter (Promega, Madison, WI, USA), respectively $(5 x(d N R 1)-5 x(e N R 3 A 4)-p G L 3)$. The cDNA of mPXR was amplified by PCR and inserted into pTargeT (Promega). phRL-TK (Promega) was used as control plasmid to normalize transfection efficacy.

\section{Reporter assay}

HepG2 cells (RIKEN BioResource Center, Ibaraki, Japan) were seeded on 96-well plates (Nest Biotechnology, Wuxi, China) at 10,000 cells/well. The cells were transfected with $5 x(\mathrm{dNR} 1)-5 \mathrm{x}(\mathrm{eNR} 3 \mathrm{~A} 4)$ pGL3, mPXR expression plasmid and phRL-TK with JetPEI (Polyplus transfection, Illkirch, France). Twenty-four hours after transfection, cells were treated with food additives $(3,10$ or $30 \mu \mathrm{M}), 10 \mu \mathrm{M}$ PCN or vehicle (final concentration of $0.1 \%$ dimethyl sulfoxide (DMSO)) for $24 \mathrm{hr}$. The cell lysates were subjected to Dual Luciferase Assay System (Promega). Firefly luciferase activity was normalized to Renilla luciferase activity.

\section{Animal treatment}

All experiments were performed in accordance with the guidelines for animal experiments of University of Shizuoka. Male C57BL/6N mice (Charles River Japan, Kanagawa, Japan) were maintained in a temperatureand light-controlled environment $\left(24^{\circ} \mathrm{C}, 12\right.$-hr light/dark cycle). Seven to nine weeks old mice were used for the experiments. Mice were intraperitoneally treated with imazalil (100 mg/kg), PCN (100 mg/kg) or vehicle (corn oil, $20 \mathrm{~mL} / \mathrm{kg})$. Twenty-four hours after the treatment, mice were sacrificed by cervical dislocation and the liv- 
Imazalil enhances hepatocyte proliferation through PXR activation

ers were collected. To test the dose-dependency of imazalil, mice were intraperitoneally treated with imazalil $(25,50,75$ or $100 \mathrm{mg} / \mathrm{kg})$, PCN (100 mg/kg), TCPOBOP (3 $\mathrm{mg} / \mathrm{kg}$ ) or vehicle (corn oil, $20 \mathrm{~mL} / \mathrm{kg}$ ). Forty-eight hours after the treatment, mice were sacrificed by cervical dislocation and the livers were collected. For the assessment with co-exposure to imazalil on CAR activator treatment, mice were intraperitoneally treated with vehicle (corn oil, $20 \mathrm{~mL} / \mathrm{kg}$ ), TCPOBOP (3 mg/kg), imazalil (75 mg/kg) or both of imazalil $(75 \mathrm{mg} / \mathrm{kg})$ and TCPOBOP $(3 \mathrm{mg} / \mathrm{kg})$. Forty-eight hours after the treatment, mice were sacrificed by cervical dislocation and their livers were collected.

\section{Determination of mRNA levels}

Total RNA isolation and cDNA synthesis were carried out as described previously (Shizu et al., 2016). Quantitative RT-PCR (qRT-PCR) was performed using GoTaq qPCR Master Mix (Promega) and primer pairs for genes of interest shown in Supplementary Table S1. Target mRNA levels were normalized by Actb mRNA levels.

\section{Immunohistochemistry}

Livers were fixed in 10\% neutral buffered formalin (Wako Pure Chemical Industries). Paraffin-embedded livers were sectioned at 4- $\mu \mathrm{m}$ thickness and the sections were stained with anti-Ki-67 antibody and counter stained with hematoxylin using standard procedures by Morphotechnology (Hokkaido, Japan). Image capture and acquisition were carried out with DMI1/MC120 (Leica Microsystems, Wetzlar, Germany). Image J software (National Institutes of Health, Bethesda, MA, USA) was used for the analysis of data. The proliferation index was determined as follows: total and Ki-67-positive nuclei were counted in randomly selected three areas (magnification; $\mathrm{x} 25$ ) per each section from individual mouse and the percentage of Ki-67-positive nuclei for each mouse was calculated. Then, the mean and standard deviation (S.D.) values for each experimental group were calculated.

\section{Statistical analysis}

Statistical analysis was performed using JMP Pro 12 (SAS Institute, Cary, NC, USA). All data are provided as the means \pm S.D. The significance of difference between control and treated groups was assessed using Student's t-test (between two groups), ANOVA followed by Dunnett's test (between control and multiple treated groups) or Tukey-Kramer test (for multiple groups).

\section{RESULTS}

\section{Screening of food additives for MPXR activation by reporter assays}

We screened food additives that are highly used in Japan and the related compounds shown in Table 1 for their ability to activate mPXR using an in vitro reporter assay. HepG2 cells were transfected with the reporter plasmid (5x(dNR1)-5x(eNR3A4)-pGL3) and expression plasmid for mPXR (mPXR-pTarget) and treated with the 25 test compounds at 3,10 or $30 \mu \mathrm{M}$ or the typical mPXR ligand PCN. PCN treatment induced reporter activities 7.7-fold against vehicle-treated group. Table 1 shows the relative reporter activities to those in PCN-treated cells, whose increase was set at $100 \%$. Among the test compounds, imazalil (Fig. 1A) strongly activated mPXR and increased reporter activities by more than $80 \%$. To confirm mPXR activation by imazalil, we conducted report-

A<smiles>C=CCOC(Cn1ccnc1)c1ccc(Cl)cc1Cl</smiles>

B

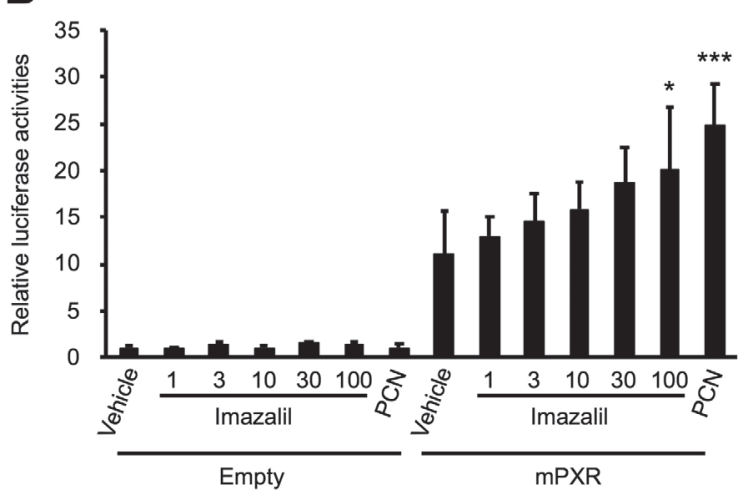

Fig. 1. Dose-dependent activation of mPXR by imazalil in HepG2 cells. A. Chemical structure of imazalil. B. HepG2 cells transfected with $5 x(\mathrm{dNR} 1)-5 \mathrm{x}(\mathrm{eNR} 3 \mathrm{~A} 4)-$ pGL3, phRL-TK, empty- or mPXR-pTarget were treated with vehicle $(0.1 \%$ DMSO $)$, PCN $(10 \mu \mathrm{M})$ or imazalil $(1,3,10,30$ or $100 \mu \mathrm{M})$ for $24 \mathrm{hr}$. Firefly luciferase activities were normalized to Renilla luciferase activities. Values are the mean \pm S.D. $(n=4)$. $*, \mathrm{P}<0.05$; ***, $\mathrm{P}<0.001$ (Dunnett's test versus vehicle-treated group). 
Table 1. Activation of mPXR by food additives.

\begin{tabular}{|c|c|c|c|c|c|}
\hline \multirow{2}{*}{ Name } & \multirow{2}{*}{ CAS No. } & \multirow{2}{*}{ Molecular weight } & \multicolumn{3}{|c|}{$\%$ activation } \\
\hline & & & $3 \mu \mathrm{M}$ & $10 \mu \mathrm{M}$ & $30 \mu \mathrm{M}$ \\
\hline Acesulfame K & $55589-62-3$ & 201.24 & $26.8 \pm 12.8$ & $17.5 \pm 2.5$ & $21.2 \pm 4.5$ \\
\hline Annatto & $1393-63-1$ & - & $20.7 \pm 9.1$ & $21.7 \pm 5.0$ & $17.5 \pm 4.8$ \\
\hline $\mathrm{L}(+)$-Ascorbic acid & $50-81-7$ & 176.12 & $10.8 \pm 2.2$ & $12.8 \pm 5.2$ & $14.9 \pm 8.2$ \\
\hline Aspartame & $22839-47-0$ & 294.31 & $21.3 \pm 8.1$ & $24.1 \pm 4.8$ & $28.4 \pm 11.2$ \\
\hline Benzoic acid & $65-85-0$ & 122.12 & $10.1 \pm 2.4$ & $12.0 \pm 1.8$ & $19.2 \pm 10.3$ \\
\hline Dibutylhydroxytoluene & $128-37-0$ & 220.35 & $11.0 \pm 2.9$ & $10.1 \pm 1.9$ & $14.8 \pm 4.3$ \\
\hline Food Blue No. 1 & $3844-45-9$ & 792.84 & $33.1 \pm 4.8$ & $26.9 \pm 5.4$ & $36.1 \pm 17.6$ \\
\hline Food Red No. 3 & $16423-68-0$ & 879.86 & $37.4 \pm 17.7$ & $30.2 \pm 13.5$ & $20.2 \pm 9.3$ \\
\hline Food Red No. 40 & $25956-17-6$ & 496.42 & $35.1 \pm 12.8$ & $17.9 \pm 4.6$ & $41.7 \pm 9.3$ \\
\hline Food Red No. 106 & $3520-42-1$ & 580.65 & $22.5 \pm 8.5$ & $30.1 \pm 5.5$ & $26.2 \pm 4.1$ \\
\hline Food Red No. 102 & $2611-82-7$ & 604.46 & $29.0 \pm 6.8$ & $41.3 \pm 9.9$ & $22.6 \pm 12.1$ \\
\hline Food Yellow No. 4 & $1934-21-0$ & 534.36 & $24.0 \pm 4.9$ & $26.3 \pm 8.4$ & $28.1 \pm 6.8$ \\
\hline Food Yellow No. 5 & 2783-94-0 & 452.36 & $32.1 \pm 9.9$ & $28.5 \pm 10.3$ & $23.7 \pm 3.3$ \\
\hline Glycyrrhizic acid monoammonium salt n-hydrate & $53956-04-0$ & 839.96 & $23.7 \pm 6.4$ & $24.7 \pm 6.9$ & $21.6 \pm 8.2$ \\
\hline Imazalil & $35554-44-0$ & 297.18 & $20.4 \pm 4.3$ & $31.5 \pm 9.4$ & $80.4 \pm 17.4$ \\
\hline D(-)-Isoascorbic acid & $89-65-6$ & 176.12 & $9.5 \pm 2.0$ & $12.1 \pm 4.3$ & $12.1 \pm 3.3$ \\
\hline Propylene glycol & $57-55-6$ & 76.09 & $23.0 \pm 11.3$ & $23.1 \pm 3.3$ & $20.6 \pm 5.9$ \\
\hline Rebaudioside A & $58543-16-1$ & 967.02 & $24.3 \pm 9.3$ & $27.0 \pm 10.8$ & $21.0 \pm 6.1$ \\
\hline Saccharin sodium dihydrate & $6155-57-3$ & 241.20 & $23.3 \pm 6.0$ & $23.1 \pm 5.8$ & $20.6 \pm 4.1$ \\
\hline Sorbic acid & $110-44-1$ & 112.13 & $13.9 \pm 1.9$ & $13.5 \pm 4.9$ & $16.0 \pm 7.4$ \\
\hline Stevioside & $57817-89-7$ & 804.88 & $26.0 \pm 10.5$ & $15.0 \pm 6.2$ & $31.5 \pm 13.1$ \\
\hline Sucralose & $56038-13-2$ & 397.63 & $21.5 \pm 5.6$ & $28.2 \pm 10.9$ & $20.0 \pm 8.8$ \\
\hline Thiabendazole & $148-79-8$ & 201.25 & $15.7 \pm 1.9$ & $22.2 \pm 4.9$ & $26.1 \pm 7.4$ \\
\hline$( \pm)$ - $\alpha$-Tocopherol & $10191-41-0$ & 430.71 & $28.8 \pm 9.5$ & $36.7 \pm 23.9$ & $22.3 \pm 9.0$ \\
\hline$(+)-\gamma$-Tocopherol & $54-28-4$ & 416.68 & $26.0 \pm 7.4$ & $18.5 \pm 6.3$ & $27.9 \pm 13.8$ \\
\hline
\end{tabular}

Reporter assays were performed as described in MATERIALS AND METHODS. Cells were treated with vehicle, test compounds (3, 10 or $30 \mu \mathrm{M})$ or PCN $(10 \mu \mathrm{M}$, a positive control) for $24 \mathrm{hr}$. Reporter activities of cells treated with each food additive are shown as \% activation with those in the cells treated with PCN being set as $100 \%$. Values are the means \pm S.D. $(n=4)$.

er assays with the same reporter plasmid in the presence or absence of mPXR expression plasmid. As results, imazalil treatment dose-dependently induced the reporter gene expression only in mPXR-expressed HepG2 cells (Fig. 1B). Therefore, we selected this compound as a mPXR activator candidate for further experiments.

\section{mPXR activation by imazalil in mouse livers}

To investigate the influence of imazalil treatment on PXR activation in vivo, male C57BL/6 mice were intraperitoneally treated with $100 \mathrm{mg} / \mathrm{kg}$ imazalil for $24 \mathrm{hr}$ and hepatic mRNA levels of PXR target genes (Cyp3a11 and Cyp2b10) were determined. As shown in Fig. 2A, imazalil treatment significantly increased Cyp3a11 and Cyp2b10 mRNA levels more than PCN treatment, suggesting that imazalil strongly activates PXR in mouse livers. To confirm MPXR activation by imazalil, male mice were treated with several doses of imazalil as well as PCN and the mouse CAR (mCAR) agonist TCPOBOP, for $48 \mathrm{hr}$ and hepatic mRNA levels of Cyp3a11 and Cyp2b10 were determined (Fig. 2B). Hepatic Cyp3all mRNA levels were significantly increased by imazalil in a dosedependent manner as expected. In contrast, no significant increase in the hepatic Cyp2b10 mRNA levels was observed with all the doses for imazalil at $48 \mathrm{hr}$ although there was a tendency to increase with $100 \mathrm{mg} / \mathrm{kg}$ imazalil. Under this condition, TCPOBOP treatment increased the mRNA levels around 700-fold. Cyp3a11 and Cyp2b10 are common target genes of mPXR and mCAR. Thus, to confirm that the imazalil-induced expression of Cyp $3 a 11$ and Cyp $2 b 10$ were mediated by mPXR, the ratio of Cyp $2 b 10$ and Cyp3a11 mRNA levels were calculated (Fig. 2C). The ratios of imazalil at 25,50 and $75 \mathrm{mg} / \mathrm{kg}$ were almost same as that of PCN. While the ratio of $100 \mathrm{mg} / \mathrm{kg}$ imazalil was slightly higher than that of PCN, it was substantially smaller than that of TСРОВOP. These results suggest that imazalil activates $\mathrm{mPXR}$ but not MCAR in mouse livers at least at the doses used. 
Imazalil enhances hepatocyte proliferation through PXR activation

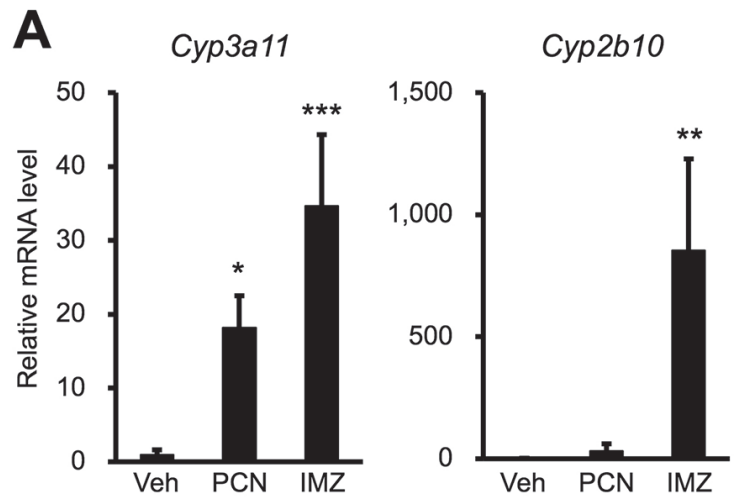

B

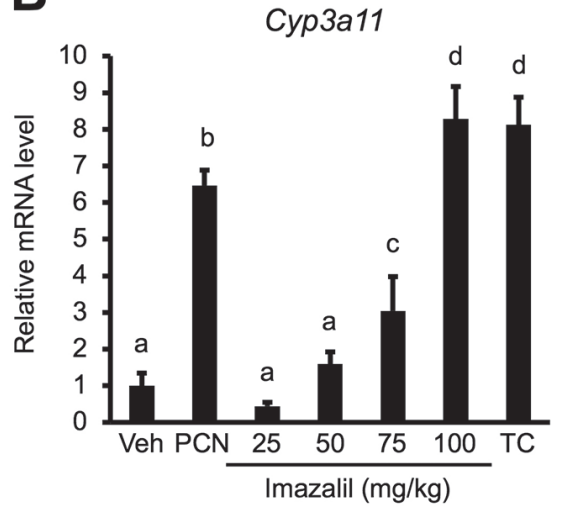

Cyp2b10

C
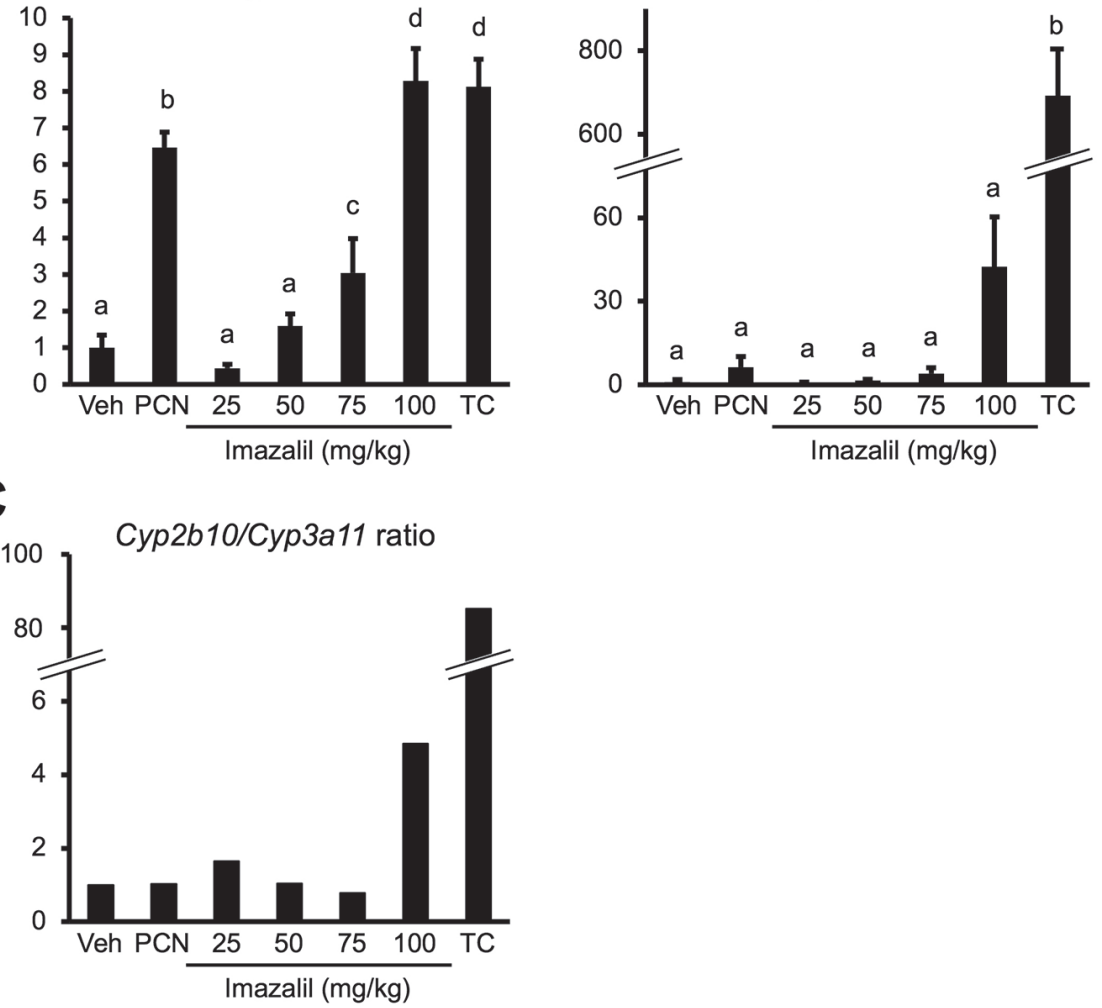

Fig. 2. mPXR activation by imazalil treatment in mouse livers. A. Male mice were treated intraperitoneally with vehicle (corn oil; Veh), PCN $(100 \mathrm{mg} / \mathrm{kg}$ ) or imazalil (IMZ, $100 \mathrm{mg} / \mathrm{kg}$ ) for $24 \mathrm{hr}$. Total hepatic RNAs were subjected to qRT-PCR for Cyp3all and Cyp2b10. Values are the mean \pm S.D. $(\mathrm{n}=3-4)$. *, $\mathrm{P}<0.05 ;{ }^{* *}, \mathrm{P}<0.01 ; * * *, \mathrm{P}<0.001$ (Dunnett's test, versus vehicletreated group). B. Male mice were treated intraperitoneally with vehicle (corn oil; Veh), PCN (100 mg/kg), TCPOBOP (TC, $3 \mathrm{mg} / \mathrm{kg})$ or imazalil $(25,50,75$ or $100 \mathrm{mg} / \mathrm{kg})$ for $48 \mathrm{hr}$. Total hepatic RNAs were subjected to qRT-PCR for Cyp3a11 and Cyp2b10. Values are the mean \pm S.D. $(\mathrm{n}=3-5)$. Columns not sharing a common letter $(\mathrm{a}, \mathrm{b}, \mathrm{c}$ and d) differ significantly with each other ( $\mathrm{P}<0.05$; Tukey-Kramer test). Ratios of Cyp2b10 mRNA levels to Cyp3a11 mRNA levels were calculated in C.

Influence of imazalil on the hepatocyte proliferation mediated by CAR activation

We finally investigated whether imazalil treatment could accelerate the hepatocyte proliferation mediated by
mCAR activation. To this end, mice were treated intraperitoneally with TCPOBOP in combination with or without imazalil $(75 \mathrm{mg} / \mathrm{kg})$ for $48 \mathrm{hr}$. Relative liver weights were increased by single treatment with imazalil or 
A

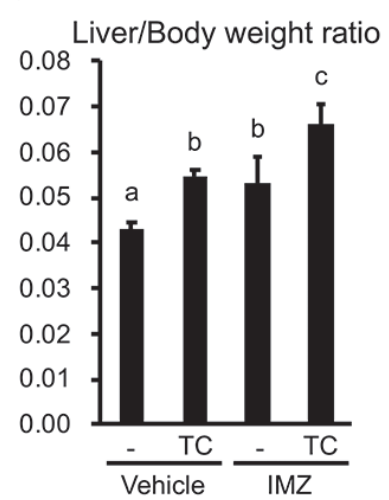

B

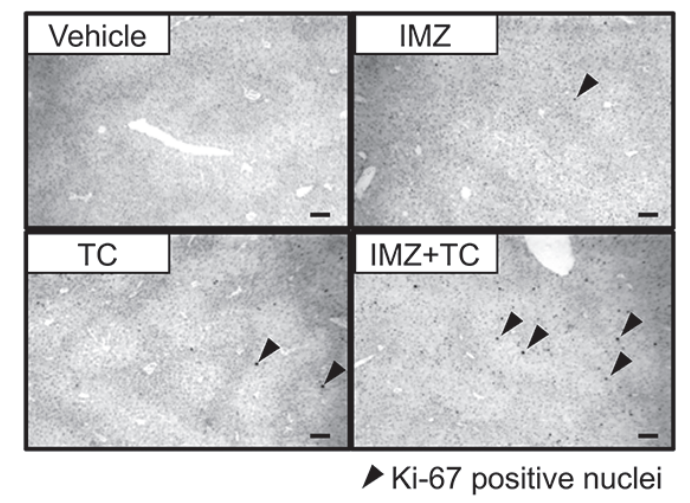

C

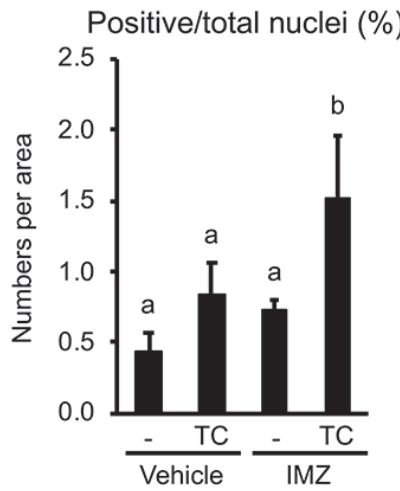

D

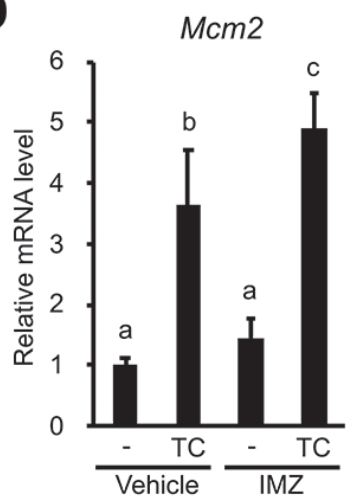

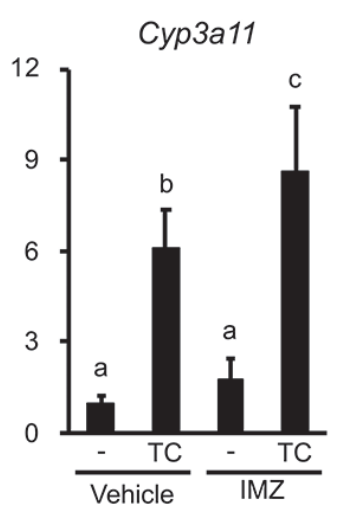

Cyp2b10

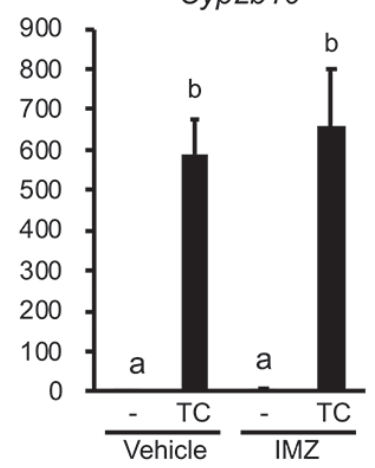

Fig. 3. Influence of imazalil treatment on hepatocyte proliferation in mice. Male mice were treated intraperitoneally with vehicle (corn oil), TCPOBOP (TC, $3 \mathrm{mg} / \mathrm{kg}$ ), imazalil (IMZ, $75 \mathrm{mg} / \mathrm{kg}$ ) or both TCPOBOP and imazalil for $48 \mathrm{hr}$. A. The liver to body weight ratios were calculated. B. Livers were fixed and liver sections were stained with anti-Ki-67 antibody. Arrowheads indicate Ki-67-positive nuclei. Scale bar, $100 \mu \mathrm{m}$. C. Total and Ki-67-positive nuclei were counted in three randomly selected areas per each section. D. Total hepatic RNAs were subjected to qRT-PCR for Mcm2, Cyp3a11 and Cyp2b10. Values are the mean \pm S.D. $(n=4$ or 5$)$. Columns not sharing a common letter $(a, b$ and $c)$ differ significantly with each other $(\mathrm{P}<0.05$; Tukey-Kramer test).

TCPOBOP and co-treatment with both further increased them (Fig. 3A).

Immunostaining of liver sections with $\mathrm{Ki}-67$, a cell proliferation marker protein, demonstrated that single TCPOBOP or imazalil treatment tended to increase the number of Ki-67-positive nuclei (Figs. 3B, 3C). Cotreatment with imazalil and TCPOBOP significantly increased the number of positive nuclei compared to single TCPOBOP treatment as expected (Figs. 3B, 3C). Hepatic mRNA levels of $M c m 2$, a cell proliferation marker gene, were increased by TCPOBOP treatment but not by imazalil treatment (Fig. 3D). In similar to Ki-67-positive nuclei, co-treatment with imazalil further increased Mcm 2 mRNA levels compared to single TCPOBOP treat- ment with a statistical significance (Fig. 3D). mCAR activation by TCPOBOP treatment was confirmed by significant increases in hepatic Cyp2b10 and Cyp3a11 mRNA levels (Fig. 3D). These results suggest that imazalil treatment enhances hepatocyte proliferation mediated by mCAR activation in mice.

To further confirm imazalil's enhancing effect on hepatocyte proliferation, mice were treated with $75 \mathrm{mg} / \mathrm{kg}$ imazalil twice (once a day) with or without TCPOBOP and $24 \mathrm{hr}$ after the last treatment livers were subjected to the investigation of hepatocyte proliferation (Supplementary Fig. S1). Repeated treatment with imazalil did not increase the number of Ki-67-positive nuclei and $\mathrm{Mcm} 2$ mRNA levels as in Fig. 3. However, combined treatment 
Imazalil enhances hepatocyte proliferation through PXR activation

with imazalil and TCPOBOP much greatly increased them compared to its single treatment. The results confirmed that imazalil treatment accelerated hepatocyte proliferation mediated by TCPOBOP treatment in mice.

\section{DISCUSSION}

Screening of food additives and the associated compounds for $\mathrm{mPXR}$ activation with reporter assays in HepG2 cells identified imazalil as a strong mPXR activator. MPXR activation by imazalil was confirmed in vivo by treating mice with imazalil and the determination of hepatic mRNA levels of mPXR target genes Cyp3a11 and Cyp2b10. Comparison of imazalil with the strong mPXR ligand PCN and mCAR ligand TCPOBOP demonstrated that the inducing profile of hepatic Cyp3a11 and Cyp $2 b 10$ mRNA levels by imazalil was very similar to that by PCN but not TCPOBOP. These results suggest that imazalil activates $\mathrm{mPXR}$ and hardly activate $\mathrm{mCAR}$ at least at the doses of 25 to $75 \mathrm{mg} / \mathrm{kg}$. Recently, imazalil feeding of CAR knockout mice was reported to increase hepatic Cyp $2 b 10 \mathrm{mRNA}$ levels as in wild-type mice (Tamura et al., 2016). It supports our hypothesis that in vivo in mouse livers imazalil activates mPXR and its mCAR activation is negligible.

Recently, we have demonstrated that PXR activation accelerates CAR-dependent as well as growth factor-mediated proliferation of murine hepatocytes by increasing the sensitivity to proliferation stimuli through the downregulation of cyclin-dependent kinase inhibitor proteins (Shizu et al., 2013, 2016). We thus co-treated mice with TCPOBOP and imazalil to verify whether imazalil treatment could accelerate hepatocyte proliferation induced by TCPOBOP through $\mathrm{mPXR}$ activation. Single or 2-day treatment with imazalil of $75 \mathrm{mg} / \mathrm{kg}$ alone did not affect hepatocyte proliferation determined by Ki-67 staining and Mcm 2 mRNA levels. However, co-treatment with imazalil and TCPOBOP increased the number of hepatic Ki-67 -positive nuclei and Mcm 2 mRNA levels more greatly than single TCPOBOP treatment. These results suggest that imazalil treatment enhances mCAR-dependent hepatocyte proliferation through PXR activation. Accordingly, co-exposure to imazalil with liver tumor promoters such as CAR activators may increase the risk of liver cancer formation.

Our present results indicate that imazalil did not activate CAR in mouse livers at the doses we used. However, long-term treatment with 500 ppm imazalil alone was reported to increase the incidence of eosinophilic adenomas in the liver of wild-type but not in CAR knockout mice (Tamura et al., 2016). Since $100 \mathrm{mg} / \mathrm{kg}$ ima- zalil treatment slightly increased the ratio of hepatic Cyp2b10/Cyp3a11 mRNA levels, a high dose of imazalil might activate CAR as well as PXR in mouse livers. Thus, in terms of liver tumor formation, imazalil may play dual roles through activating PXR to enhance hepatocyte proliferation induced by various stimuli, including CAR activation and growth factors, and directly activating CAR at a high dose.

There are large species differences between mouse and human PXR activators because of the structural differences in the ligand binding pockets of mouse and human PXR (LeCluyse, 2001). However, some chemicals such as 5 $\beta$-pregnane-3,20-dione, mevastatin and nifedipine are reported to be common ligands for mouse and human PXR (Jones et al., 2000; Moore et al., 2003). In our preliminary experiments, imazalil also activated human PXR in reporter assays (unpublished results). In addition, a previous report suggested that imazalil activated human PXR (Lemaire et al., 2006). These results suggest that imazalil is a common ligand for mouse and human PXR. Further experiments are required to characterize imazalil as a PXR activator common to mouse and human, and the influence of imazalil intake in humans.

Imazalil, also known as enilconazole, is an imidazolecontaining anti-mold agent, and is approved as a food additive in Japan and is often used as a post-harvest agent for citruses, bananas or other fruits worldwide. Imazalil inhibits the sterol biosynthesis of fungi through inhibiting $14 \alpha$-demethylase (Hargrove et al., 2017). Imazalil is usually left in citrus, pears and apples (Kodama et al., 2003; Donnarumma et al., 2005) and its ADI is set at $0.025 \mathrm{mg} / \mathrm{kg}$ body weight/day in Japan. Although imazalil may activate human PXR as discussed above, it is unlikely that residual imazalil in food activates PXR in human livers considering the amount of imazalil taken with food as long as it is used within the ADI. However, pesticides can be contained in foods more than its maximum residue limit level in some countries (Fernández et al., 2001; Ortelli et al., 2005). These excessive amounts of pesticide residues increase a chance to activate PXR in human livers, resulting in unusual metabolisms of xenobiotics through the induction of drug-metabolizing enzymes, and possible adverse events associated with PXR activation. Therefore, the assessment of food additives for their abilities to activate PXR and other nuclear receptors might be useful for their safety use.

In conclusion, the present results suggest that imazalil is a mPXR activator and can enhance hepatocyte proliferation mediated by CAR-activating tumor promoters through PXR activation when administered in combination. Generally, current chemical toxicity tests evaluate 
the toxicity caused by a single chemical compound. Our results imply the importance of safety evaluation considering the simultaneous exposure to multiple substances as we intake several types of xenobiotics, such as drugs, foods, supplements, food additives, pesticide residues or industrial chemicals, intentionally and unintentionally in our daily life.

\section{ACKNOWLEDGMENTS}

This work was supported by a grant from the Japan Food Chemical Research Foundation and JSPS KAKENHI Grant Number 17K08418.

Conflict of interest---- The authors declare that there is no conflict of interest.

\section{REFERENCES}

Chang, T.K. and Waxman, D.J. (2006): Synthetic drugs and natural products as modulators of constitutive androstane receptor (CAR) and pregnane X receptor (PXR). Drug Metab. Rev., 38, 51-73.

Donnarumma, L., Pompi, V., Rossi, E. and Carfî, F. (2005): Fungicide residues in pears and apples after post harvest treatments by drencher. Commun. Agric. Appl. Biol. Sci., 70, 1053-1058.

Dusek, J., Carazo, A., Trejtnar, F., Hyrsova, L., Holas, O., Smutny, T., Micuda, S. and Pavek, P. (2017): Steviol, an aglycone of steviol glycoside sweeteners, interacts with the pregnane X (PXR) and aryl hydrocarbon (AHR) receptors in detoxification regulation. Food Chem. Toxicol., 109, 130-142.

Fernández, M., Picó, Y. and Mañes, J. (2001): Pesticide residues in oranges from Valencia (Spain). Food Addit. Contam., 18, 615624.

Goodwin, B., Hodgson, E. and Liddle, C. (1999): The orphan human pregnane $\mathrm{X}$ receptor mediates the transcriptional activation of CYP3A4 by rifampicin through a distal enhancer module. Mol. Pharmacol., 56, 1329-1339.

Gotoh, S. and Negishi, M. (2015): Statin-activated nuclear receptor PXR promotes SGK2 dephosphorylation by scaffolding PP2C to induce hepatic gluconeogenesis. Sci. Rep., 5, 14076.

Hargrove, T.Y., Friggeri, L., Wawrzak, Z., Qi, A., Hoekstra, W.J., Schotzinger, R.J., York, J.D., Guengerich, F.P. and Lepesheva, G.I. (2017): Structural analyses of Candida albicans sterol $14 \alpha-$ demethylase complexed with azole drugs address the molecular basis of azole-mediated inhibition of fungal sterol biosynthesis. J. Biol. Chem., 292, 6728-6743.

Hukkanen, J. (2012): Induction of cytochrome P450 enzymes: a view on human in vivo findings. Expert Rev. Clin. Pharmacol., 5, 569-585.

Jones, S.A., Moore, L.B., Shenk, J.L., Wisely, G.B., Hamilton, G.A., McKee, D.D., Tomkinson, N.C., LeCluyse, E.L., Lambert, M.H., Willson, T.M., Kliewer, S.A. and Moore, J.T. (2000): The pregnane $\mathrm{X}$ receptor: a promiscuous xenobiotic receptor that has diverged during evolution. Mol. Endocrinol., 14, 27-39.

Kodama, S., Yamamoto, A., Ohura, T., Matsunaga, A. and Kanbe, T. (2003): Enantioseparation of imazalil residue in orange by capillary electrophoresis with 2-hydroxypropyl-beta-cyclodextrin as a chiral selector. J. Agric. Food Chem., 51, 6128-6131.

Lau, K., McLean, W.G., Williams, D.P. and Howard, C.V. (2006): Synergistic interactions between commonly used food additives in a developmental neurotoxicity test. Toxicol. Sci., 90, 178187.

LeCluyse, E.L. (2001): Pregnane X receptor: molecular basis for species differences in CYP3A induction by xenobiotics. Chem. Biol. Interact., 134, 283-289.

Lemaire, G., Mnif, W., Pascussi, J.M., Pillon, A., Rabenoelina, F., Fenet, H., Gomez, E., Casellas, C., Nicolas, J.C., Cavaillès, V., Duchesne, M.J. and Balaguer, P. (2006): Identification of new human pregnane $\mathrm{X}$ receptor ligands among pesticides using a stable reporter cell system. Toxicol. Sci., 91, 501-509.

Moore, J.T., Moore, L.B., Maglich, J.M. and Kliewer, S.A. (2003): Functional and structural comparison of PXR and CAR. Biochim. Biophys. Acta, 1619, 235-238.

Mutlu Altundağ, E., Yilmaz, A.M., Koçtürk, S., Taga, Y. and Yalçın, A.S. (2018): Synergistic Induction of Apoptosis by Quercetin and Curcumin in Chronic Myeloid Leukemia (K562) Cells. Nutr. Cancer, 70, 97-108.

Ortelli, D., Edder, P. and Corvi, C. (2005): Pesticide residues survey in citrus fruits. Food Addit. Contam., 22, 423-428.

Seow, C.L. and Lau, A.J. (2017): Differential activation of pregnane $\mathrm{X}$ receptor by carnosic acid, carnosol, ursolic acid, and rosmarinic acid. Pharmacol. Res., 120, 23-33.

Shizu, R., Abe, T., Benoki, S., Takahashi, M., Kodama, S., Miayata, M., Matsuzawa, A. and Yoshinari, K. (2016): PXR stimulates growth factor-mediated hepatocyte proliferation by cross-talk with the FOXO transcription factor. Biochem. J., 473, 257-266.

Shizu, R., Benoki, S., Numakura, Y., Kodama, S., Miyata, M., Yamazoe, Y. and Yoshinari, K. (2013): Xenobiotic-induced hepatocyte proliferation associated with constitutive active/ androstane receptor (CAR) or peroxisome proliferator-activated receptor $\alpha(\mathrm{PPAR} \alpha)$ is enhanced by pregnane $\mathrm{X}$ receptor (PXR) activation in mice. PLoS One, 8, e61802.

Spruiell, K., Richardson, R.M., Cullen, J.M., Awumey, E.M., Gonzalez, F.J. and Gyamfi, M.A. (2014): Role of pregnane X receptor in obesity and glucose homeostasis in male mice. J. Biol. Chem., 289, 3244-3261.

Staudinger, J.L., Goodwin, B., Jones, S.A., Hawkins-Brown, D., MacKenzie, K.I., LaTour, A., Liu, Y., Klaassen, C.D., Brown, K.K., Reinhard, J., Willson, T.M., Koller, B.H. and Kliewer, S.A. (2001): The nuclear receptor PXR is a lithocholic acid sensor that protects against liver toxicity. Proc. Natl. Acad. Sci. USA, 98, 3369-3374.

Sui, Y., Xu, J., Rios-Pilier, J. and Zhou, C. (2011): Deficiency of PXR decreases atherosclerosis in apoE-deficient mice. J. Lipid Res., 52, 1652-1659.

Tamura, K., Inoue, K., Takahashi, M., Matsuo, S., Kodama, Y. and Yoshida, M. (2016): A crucial role of constitutive androstane receptor (CAR) in liver tumor development by imazalil in mice. J. Toxicol. Sci., 41, 801-811.

Toriyabe, T., Nagata, K., Takada, T., Aratsu, Y., Matsubara, T., Yoshinari, K. and Yamazoe, Y. (2009): Unveiling a new essential cis element for the transactivation of the CYP3A4 gene by xenobiotics. Mol. Pharmacol., 75, 677-684.

Wang, Y., Yuan, L., Yao, C., Ding, L., Li, C., Fang, J., Sui, K., Liu, Y. and Wu, M. (2014): A combined toxicity study of zinc oxide nanoparticles and vitamin $\mathrm{C}$ in food additives. Nanoscale, 6 , 15333-15342. 